\title{
Image-Based Biomechanical Modelling of Heart Failure
}

\author{
Martyn P. Nash
}

\begin{abstract}
Effective diagnosis and treatment of cardiovascular disease is hampered by a lack of knowledge of the underlying pathophysiological mechanisms on a patient-specific basis. Biomechanical factors, such as intrinsic myocardial stiffness and tissue stress, are known to have important influences on heart function, but these factors cannot be measured directly. Mathematical modelling provides a rational integrative basis for interpreting the rich variety of physiological data that are available in the laboratory and clinical settings. This seminar will discuss how image-based, individualised biomechanical models of the heart can be used to characterise the relative roles of anatomical, microstructural and functional remodelling in heart failure. Methods and examples from pre-clinical and clinical studies will be presented to demonstrate this approach. Individualised mathematical models of this kind can help to more specifically stratify the different forms of heart pathology, and thus have the potential to inform patient therapy and management of care.
\end{abstract}

\footnotetext{
M. P. Nash $(\bowtie)$

Auckland Bioengineering Institute, The University of Auckland, Auckland, New Zealand e-mail: martyn.nash@auckland.ac.nz
} 